\title{
Design and Comparison of the Handling Performance of Different Electric Vehicle Layouts
}

Leonardo De Novellis, Aldo Sorniotti*, Patrick Gruber

Department of Mechanical Engineering Sciences, Faculty of Engineering and Physical Sciences, University of Surrey, Guildford, United Kingdom

*Corresponding author: Department of Mechanical Engineering Sciences, Faculty of Engineering and Physical Sciences, University of Surrey, Guildford, GU2 7XH, UK. email: a.sorniotti@ surrey.ac.uk 


\section{Design and Comparison of the Handling Performance of Different Electric Vehicle Layouts}

In contrast to conventional internal combustion engine driven vehicles, the number of motors in fully electric cars is not fixed. A variety of architectural solutions, including from one to four individually controlled electric drive units, is possible and opens up new avenues in the design of vehicle characteristics. In particular, individual control of multiple electric powertrains promises to enhance handling performance in steady-state and dynamic conditions. For the analysis and the selection of the best electric powertrain layout based on expected vehicle characteristics and performance, new analytical tools and metrics are required. This article presents and demonstrates a novel offline procedure for the design of the feedforward control action of the vehicle dynamics controller of a fully electric vehicle and three performance indicators for the objective comparison of the handling potential of alternative electric powertrain layouts. The results demonstrate that the proposed offline routine allows achieving desired understeer characteristics with any of the investigated vehicle configurations, in traction and braking conditions.

With respect to linear handling characteristics, the simulations indicate that the influence of torque-vectoring is independent of the location of the controlled axles (front or rear) and considerably affected by the number of controlled axles.

Keywords: electric vehicles; torque-vectoring; optimisation; yaw moment

\section{Introduction}

In recent years significant improvements have been accomplished in the design of energy storage units and electric motors with high power density, energy density and efficiency $[1,2]$, making fully electric vehicles (FEVs) more and more a viable option for personal mobility. Current electric vehicle research is investigating different 
powertrain configurations constituted by one, two, three or four electric motors (see Figure 1) with different performance in terms of vehicle dynamics and energy saving targets $[3,4]$.
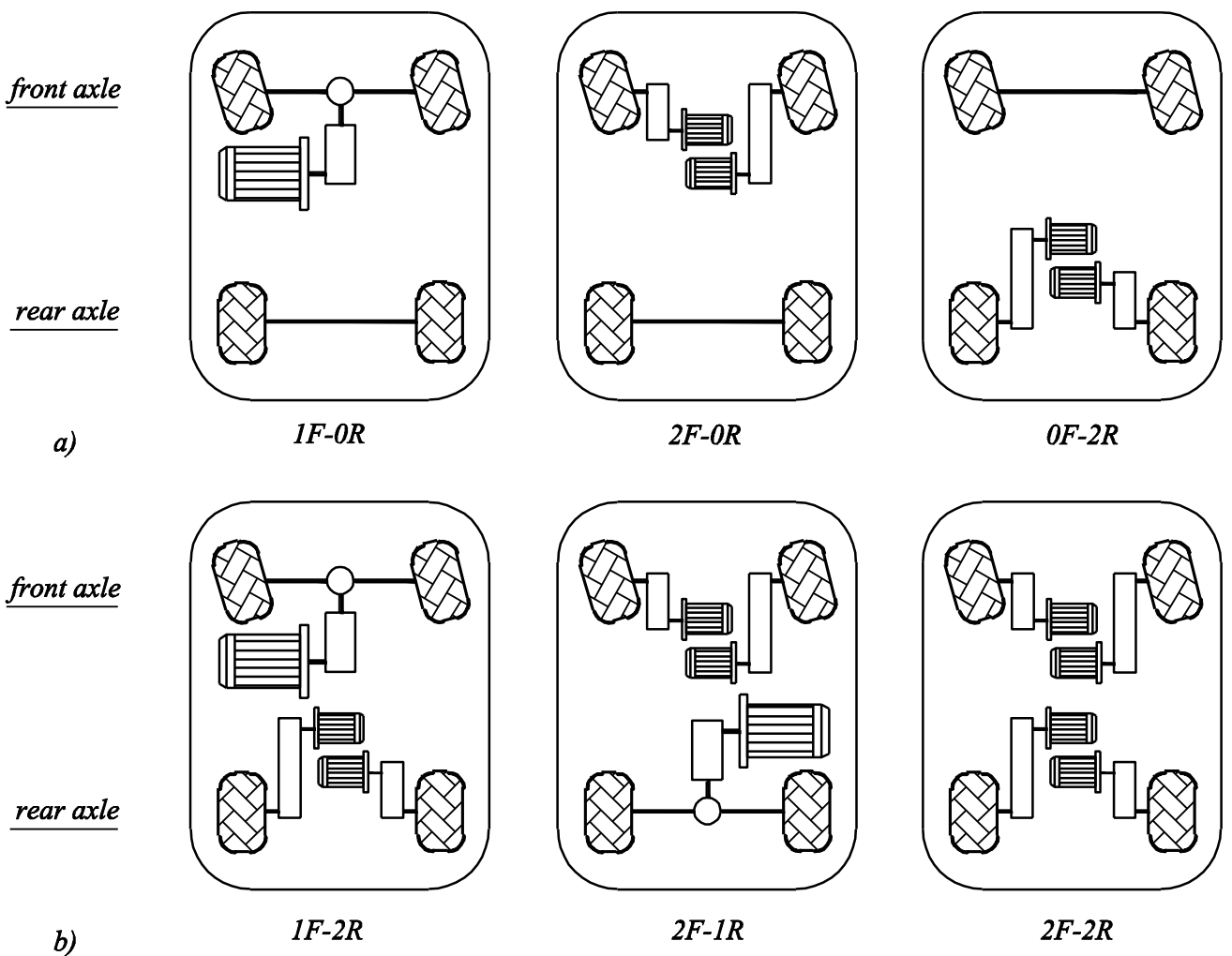

Figure 1. Examples of vehicle layouts with one to four electric powertrains. Vehicles are referred as $\mathrm{n}_{\text {mot }} \mathrm{F}-\mathrm{n}_{\text {mot }} \mathrm{R}$ where $n_{\text {mot }}$ is the number of motors on the front $(\mathrm{F})$ or rear (R) axles. In vehicles $1 \mathrm{~F}-0 \mathrm{R}, 1 \mathrm{~F}-2 \mathrm{R}$ and $2 \mathrm{~F}-1 \mathrm{R}$, the electric axle with a single motor can be equipped with a torque-vectoring differential

In relation to vehicle dynamics, FEVs have the potential to achieve hitherto impossible levels of handling qualities for road vehicles, because of the very precise controllability of electric motors. In particular, advanced motor torque modulation strategies based on the combination of front-to-rear and left-to-right wheel-torque distribution - i.e., torque-vectoring - are being developed for the implementation of 
novel yaw rate and sideslip control algorithms and for the enhancement of brake energy recuperation, anti-lock braking system (ABS) and traction control (TC) system functions $[5,6]$. The desired vehicle cornering characteristics can be designed primarily through a torque-vectoring control algorithm rather than through the traditional hardware-based chassis parameters such as mass distribution and suspension elastokinematics.

The increase in the vehicle configuration options presents also a challenge for electric vehicle designers to select the best architectural solution for specific vehicle design requirements. As recently pointed out in [7], 'despite the significant volume of theoretical studies of torque-vectoring on vehicle handling control, there is no widely accepted design methodology of how to exploit it to improve vehicle handling and stability significantly'. To address this issue, novel analytical tools and metrics are required that provide data for the engineer to make an informed design choice. In particular, specific torque-vectoring control methodologies for fully electric vehicles have to be developed, including the definition of the high-level targets of vehicle cornering response. This aspect represents one of the aims of this contribution.

In general, to avoid critical vehicle behaviour the torque-vectoring controller must be capable of continuous and smooth actuation. Current controllers adopted for conventional direct yaw moment control in production vehicles are not designed to do so as they are based on the actuation of the friction brakes when an emergency condition is detected, i.e., when the offsets between the reference and the measured (or estimated) vehicle dynamics parameters (yaw rate $r$ and sideslip angle $\beta$ ) go beyond assigned thresholds [8]. Continuous action through the integration of brake-by-wire and steer-by wire has been proposed to improve vehicle handling $[9,10]$; in these systems, the control algorithm is based on the integrated control of active front steering and 
direct yaw moment control, which can be rule-based [9] or model-based [10]. However, by relying on friction brake actuation, controller interventions will reduce vehicle speed and, thus, can be disruptive in terms of driving comfort. Also, four-wheel-steering systems $[11,12]$ allow improvements in yaw rate control, but they are capable to reduce the variation of the vehicle dynamic response induced by the longitudinal dynamics only for low values of $\beta[13]$.

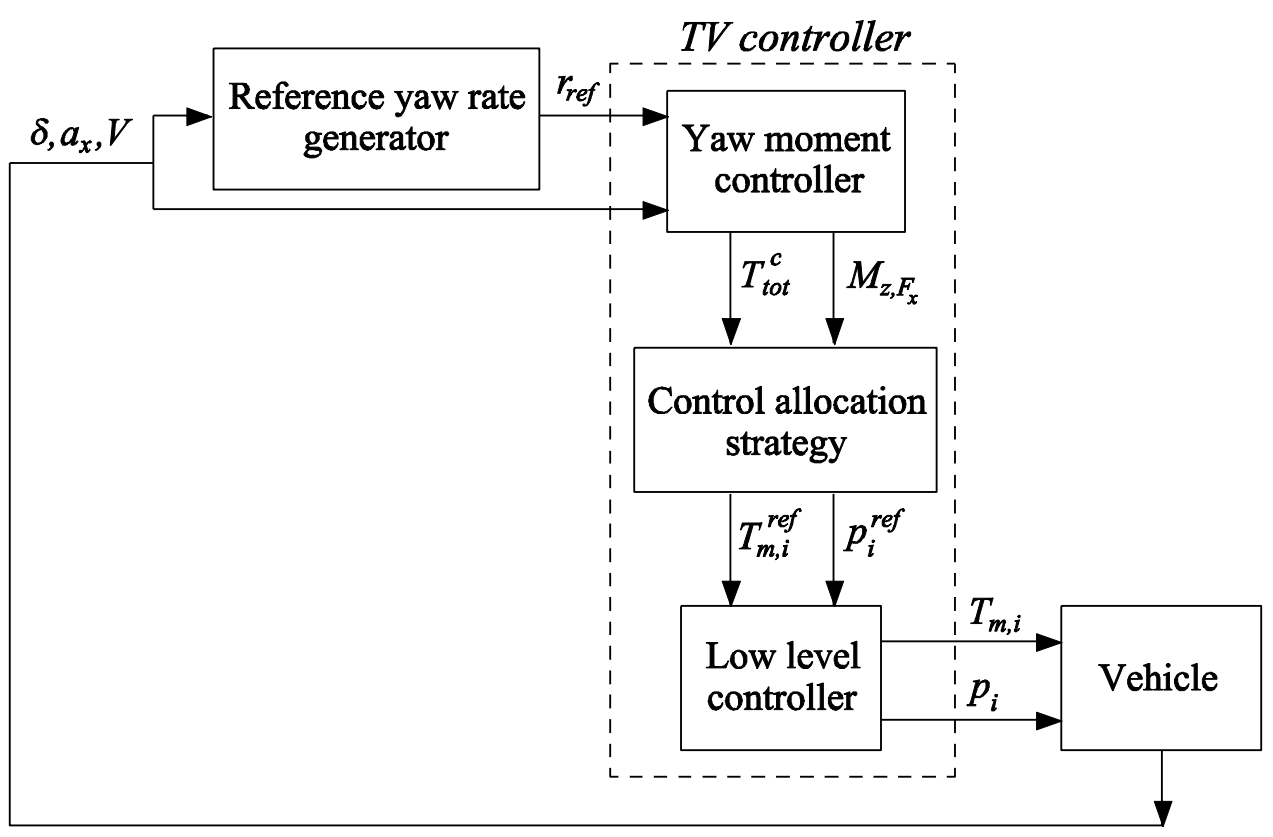

Figure 2. Control structure of the torque-vectoring algorithm for fully electric vehicles used in the simulations

Figure 2 shows the simplified block diagram of the control structure of a torquevectoring algorithm (which is also adopted here) suitable for FEVs, consisting of a reference yaw rate generator, a yaw moment controller, a wheel torque control allocation algorithm and a low level controller for individual wheel slip control. In particular, the yaw moment controller is characterised by: 
a) a feedforward part, which generates a reference yaw moment according to the vehicle dynamics objectives such as the tracking of a set of target understeer characteristics (see discussion further below).

b) a feedback part, based on the difference between a reference yaw rate (output by the reference yaw rate generator in Figure 2) and the actual yaw rate, which compensates the disturbances due to system uncertainties and transient inputs, but should provide limited contribution for low steering wheel rate conditions.

Very different and well-known control techniques can be used for the feedback yaw moment control of vehicle dynamics. For example, regulators based on the Riccati equation $[8,15]$, sliding mode controllers [16] and model predictive controllers [17] are proposed in the literature and already applied in vehicle stability control systems based on friction brake actuation. The wheel torque distribution for the achievement of the reference yaw moment (the control allocation strategy of Figure 2) can be implemented either through rule-based algorithms [18] or the application of optimal control theory [19]. In order to simulate and assess the performance of the feedback part of the torquevectoring controller, specific manoeuvres have to be simulated in the time domain [14].

In contrast, the feedforward part can be tested and, hence, designed without the need of simulations in the time domain. Instead, an optimisation procedure is required to achieve best results - which not yet exists in the literature.

This article is an account of the development of such a novel optimisation procedure for the design of the cornering response of the FEV through the offline computation of the feedforward part of the yaw moment controller and the evaluation of its actual feasibility in terms of vehicle cornering response and electric drivetrain 
characteristics. The procedure is based on an objective function formulated according to energy efficiency criteria and constrained by a reference quasi-static vehicle handling performance [11]. In addition, to complement the procedure as a powerful design tool, the paper proposes three novel performance indicators that facilitate a quick comparison of different electric vehicle architectures in terms of achievable handling characteristics.

\section{Vehicle modelling and validation}

To minimise the computational cost for running the optimisation procedure, a quasi-static vehicle model is developed and presented in section 2.1. This model is verified against results obtained with a more advanced simulation model in the time domain. To ensure accuracy of both vehicle models the simulation results are validated against experimental measurements (section 2.2).

\subsection{Vehicle models}

The optimisation procedure for the model-based derivation of the feedforward control action is applied to an 8-Degrees-of-Freedom (DoF) vehicle model. To avoid numerical forward time integration of the equations of motion, the vehicle model is based on a quasi-static approximation that assumes the yaw acceleration, $\dot{r}$, and the

sideslip rate, $\dot{\beta}$ to be zero,. The DoFs of the model are the longitudinal, lateral, roll and yaw motions of the vehicle body and the rotations of the four wheels. Assuming a flat road surface and a small vehicle sideslip angle equations (1)-(4) describe the vehicle body dynamics and Figure 3 shows the corresponding free body diagrams of the vehicle. 


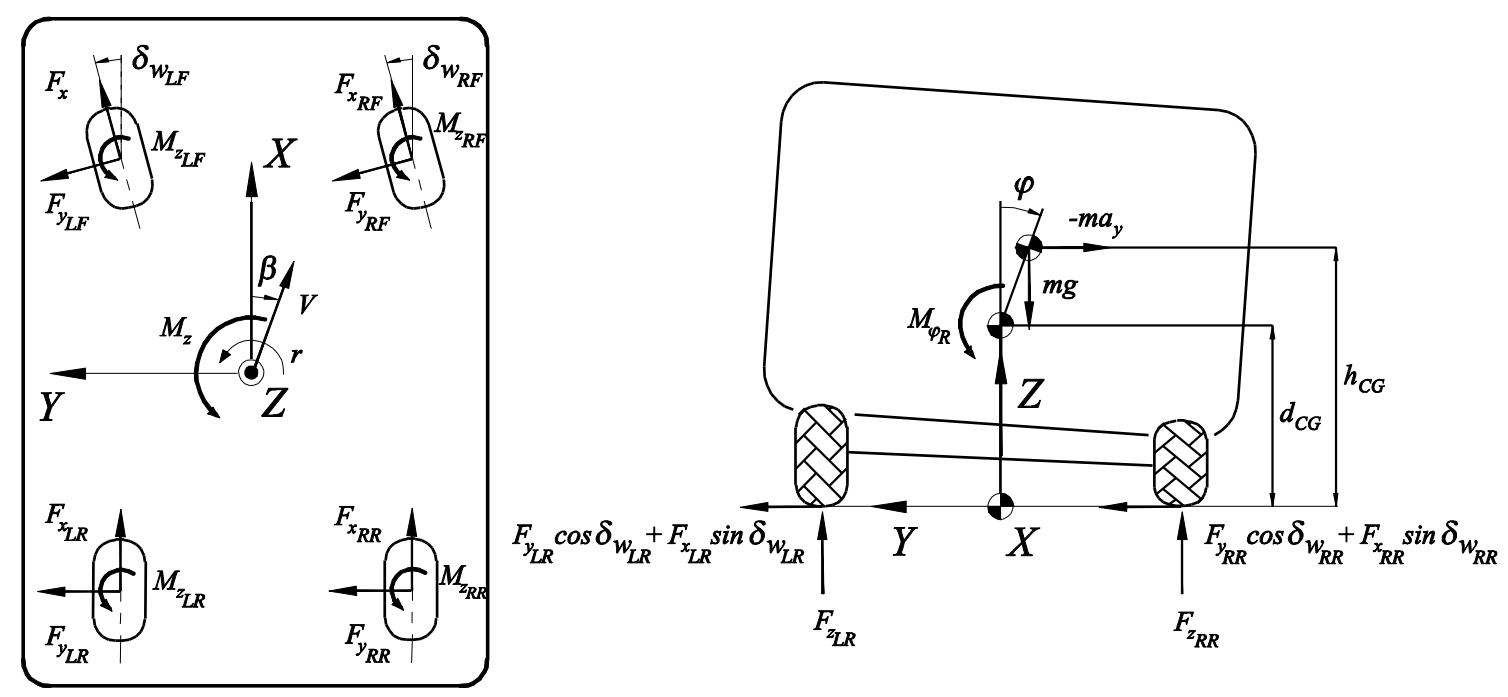

Figure 3. Top and rear views of the free body diagram of the vehicle based on the ISO vehicle reference system [22]. In the rear view (Y-Z plane), only quantities related to the rear axle have been represented, together with the vehicle roll centre at the centre of mass

Longitudinal force balance equation (neglecting equivalent mass of the wheel rotational inertia, included in the equations describing the drivetrain dynamics):

$\sum_{i=1}^{4} F_{x_{i}} \cos \delta_{w_{i}}+\sum_{i=1}^{4} F_{y_{i}} \sin \delta_{w_{i}}-F_{d r a g}=m(\dot{u}-r V \beta)$

Lateral force balance equation:

$\sum_{i=1}^{4} F_{x_{i}} \sin \delta_{w_{i}}+\sum_{i=1}^{4} F_{y_{i}} \cos \delta_{w_{i}}=m(\dot{\mathrm{V}} \beta+u r)$,

Yaw moment balance equation: 


$$
\begin{aligned}
M_{z}=\sum_{i=1}^{4} F_{x_{i}} \cos \delta_{w_{i}} y_{i}+\sum_{i=1}^{4} F_{x_{i}} \sin \delta_{w_{i}} x_{i} \\
+\sum_{i=1}^{4} F_{y_{i}} \cos \delta_{w_{i}} x_{i}+\sum_{i=1}^{4} F_{y_{i}} \sin \delta_{w_{i}} y_{i}+\sum_{i=1}^{4} M_{z_{i}}
\end{aligned}
$$

Roll moment balance equation:

$$
\begin{aligned}
m(\dot{V} \beta+u r)\left(h_{C G}\right. & \left.-d_{C G}\right) \cos \varphi+m g\left(h_{C G}-d_{C G}\right) \sin \varphi \\
& -\left(\sum_{i=1}^{2} F_{x_{i_{F}}} \sin \delta_{w_{i_{F}}}+\sum_{i=1}^{2} F_{y_{i_{F}}} \cos \delta_{w_{i_{F}}}\right)\left(d_{F}-d_{C G}\right) \\
& -\left(\sum_{i=1}^{2} F_{x_{i_{R}}} \sin \delta_{w_{i_{R}}}+\sum_{i=1}^{2} F_{y_{i_{R}}} \cos \delta_{w_{i_{R}}}\right)\left(d_{R}-d_{C G}\right)=M_{\varphi_{F}}+M_{\varphi_{R}},
\end{aligned}
$$

where the subscripts ' $F$ ' and ' $R$ ' refer to the front and rear axle respectively. $u$ is the component of the velocity of the centre of gravity of the vehicle $V$ along the longitudinal axis of the vehicle reference system. $F_{x_{i}}, F_{y_{i}}, M_{z_{i}}$ are respectively the longitudinal force, lateral force and self-aligning moment for the $i$-th tyre, evaluated in the tyre reference system, and $M_{z}$ is the yaw moment required to maintain the vehicle in trimmed conditions according to the quasi-static approach. The steering angle $\delta_{w_{i}}$ of each wheel takes into account the kinematical contributions due to the suspension and steering system design and the compliance effect induced by the load applied to the wheel. $x_{i}$ and $y_{i}$ are the longitudinal and lateral distances between each tyre and the vehicle centre of gravity; also these parameters are subject to variations depending on suspension elasto-kinematics. The height of the centre of gravity is indicated as $h_{C G}$, and $d_{C G}, d_{F}$ and $d_{R}$ are the heights of the roll axis, respectively measured at the vehicle 
centre of gravity, the front suspension and the rear suspension. The front and rear antiroll moments, $M_{\varphi_{F}}$ and $M_{\varphi_{R}}$, are expressed in the form of non-linear look-up tables taking into account only the roll stiffness contribution as the roll rate is considered to be zero $(\dot{\varphi}=0)$ in the employed quasi-static approach. $F_{d r a g}$ is the aerodynamic drag force.

The Pacejka '96 tyre model has been employed to evaluate $F_{x_{i}}, F_{y_{i}}$ and $M_{z_{i}}$ as functions of the longitudinal slip $\sigma_{i}$, slip angle $\alpha_{i}$, camber angle $\gamma_{i}$, the tyre-road friction coefficient and the vertical load $F_{z_{i}} . F_{z_{i}}$ on the $i$-th front $(F)$ or rear $(R)$ wheel is given by:

$$
\begin{aligned}
F_{z_{i}}=F_{z 0_{i}} \pm\left(F_{\text {drag }}+m(\dot{u}-r V \beta)\right) \frac{h_{C G}}{2 L} & \\
& \pm \frac{\sum_{j=1}^{2} F_{x_{j_{F / R}}} \sin \delta_{w_{j_{F / R}}}+\sum_{j=1}^{2} F_{y_{j_{F / R}}} \cos \delta_{w_{j_{F / R}}}}{\sum_{j=1}^{2}\left|y_{j_{F / R}}\right|} d_{F / R} \pm \frac{M_{\varphi_{F / R}}}{\sum_{j=1}^{2}\left|y_{j_{F / R}}\right|}
\end{aligned}
$$

where the summations $\sum_{F / R}$ are applied to the two wheels of the same axle, $F_{z 0_{i}}$ is the tyre static vertical load and $L$ is the vehicle wheelbase.

The wheel moment balance equations have the following structure:

$$
T_{i}-T_{F B_{i}}-F_{x_{i}} R_{i}-F_{z_{i}} R_{i}\left(f_{0}+f_{1} \omega_{i} R_{\text {roll }_{i}}+f_{2} \omega_{i}^{2} R_{\text {roll }_{i}}{ }^{2}\right)-J_{W_{i}} \dot{\omega}_{i}=0,
$$

where $T_{i}$ and $T_{F B_{i}}$ are respectively the electric drivetrain torque at the wheel and the friction brake torque; $R_{i}$ and $R_{\text {roll }_{i}}$ are respectively the laden radius and the rolling radius of the tyre; $J_{W_{i}}$ is the moment of inertia of the wheel. The factors $f_{0}, f_{1}$ and $f_{2}$ represent the components of the rolling resistance coefficient and $\dot{\omega}_{i}$ is the wheel angular acceleration, which is expressed as a function of the slip ratio and the velocity component $V_{x_{i}}$ of the $i$-th wheel hub along the $x$-axis of the tyre: 
$\dot{\omega}_{i}=\frac{d}{d t}\left[\frac{V_{x_{i}}}{R_{\text {roll }_{i}}}\left(\sigma_{i}+1\right)\right]=\frac{a_{x_{i}}}{R_{\text {roll }_{i}}}\left(\sigma_{i}+1\right)+\frac{V_{x_{i}}}{R_{\text {roll }_{i}}} \dot{\sigma}_{i}, \quad i=1 \ldots 4$

where the time derivative $\dot{\sigma}_{i}$ of the tyre slip ratio can be neglected according to the quasi-static approach. The set of algebraic equations (1)-(7) is completed with additional equations related to the kinematic relationships for the evaluation of the slip angles $\quad \alpha_{i}=f_{\alpha}\left(\beta, \delta_{w_{i}}, r, F_{x_{i}}, F_{y_{i}}\right) \quad$ and longitudinal slip ratios $\sigma_{i}=f_{\sigma}\left(V, \omega_{i}, \delta_{w_{i}}, r, F_{x_{i}}, F_{y_{i}}\right)$, where the explicit definition of $f_{\alpha}$ and $f_{\sigma}$, which take into account the suspension compliances, is omitted for brevity. The wheel torque distribution can be expressed as $T_{i}=f_{T_{i}}\left(T_{T O T}\right)$, where $T_{T O T}=\sum_{k=1}^{n} T_{k}$ and $f_{T_{i}}$ is the torque distribution criterion.

The difference between the alternative electric drivetrain layouts of Figure 1 is included in the dynamic equation linking the wheel torque $T_{i}$ to the electric motor torque $T_{m_{i}}$. For example, with individual wheel drivetrains consisting of an on-board electric motor drive, an on-board two-stage single-speed transmission and a half-shaft with constant velocity joints, the relevant equation reads:

$T_{m_{i}}-\frac{T_{i}}{\tau_{1_{i}} \tau_{2_{i}} \eta_{1_{i}} \eta_{2_{i}} \eta_{C V 1_{i}} \eta_{C V 2_{i}}}-J_{e q_{i}} \dot{\omega}_{m_{i}}=0$

where $\tau_{1_{i}}$ and $\tau_{2_{i}}$ are the reduction ratios of each of the two stages of the single-speed transmission; $\eta_{1_{i}}$ and $\eta_{2_{i}}$ are the equivalent efficiencies of the transmission stages; $\eta_{C V 1_{i}}$ and $\eta_{C V 2_{i}}$ are the efficiencies of the constant velocity joints located at the two sides of the half-shaft. $J_{e q_{i}}$ includes the inertial contributions due to the individual components of the drivetrain, namely: 
$J_{e q_{i}}=J_{\text {mot }_{i}}+J_{1_{i}}+\frac{J_{2_{i}}}{\tau_{1_{i}}^{2} \eta_{1_{i}}}+\frac{J_{3_{i}}}{\tau_{1_{i}}^{2} \tau_{2_{i}}^{2} \eta_{1_{i}} \eta_{2_{i}}}+\frac{J_{H S_{i}}}{\tau_{1_{i}}^{2} \tau_{2_{i}}^{2} \eta_{1_{i}} \eta_{2_{i}} \eta_{C V 1_{i}} \eta_{C V 2_{i}}}$

where $J_{1_{i}}, J_{2_{i}}$ and $J_{3_{i}}$ are the moments of inertia of the primary, secondary and output shafts of the single-speed transmission; $J_{H S_{i}}$ is the moment of inertia of the half-shaft.

For the case of a single electric motor on a driven axle (vehicles $1 \mathrm{~F}-0 \mathrm{R}, 1 \mathrm{~F}-2 \mathrm{R}$ and 2F-1R in Figure 1), the model of a torque-vectoring differential has been included, which uses multi-plate clutch packs to distribute torque between the left and right wheels. The particular model is adopted from [23], which simulates an overdriven torque-vectoring differential allowing the possibility of a torque bias also towards the faster wheel of the axle. The associated power losses are estimated from the product of differential torque output and the slip velocity of the differential clutch pack.

To calculate the input power to the electric powertrain, the electric motor drives are modelled with efficiency maps that are functions of the primary operating variables, i.e., torque, speed, input voltage and operating temperature of the motor. Also, a realistic representation of the vehicle battery is provided by a dynamic battery model that is based on the approach outlined in [24].

For verification, the results obtained with the quasi-static vehicle model are compared to those computed with a more detailed vehicle model in the time domain that has been implemented in the vehicle dynamics simulation software IPG CarMaker [25], and validated (see section 2.2). To include the six different electric powertrain layouts shown in Figure 1, a Matlab/Simulink dynamic model has been integrated in the IPG CarMaker model. With this modelling approach, the first order dynamics of the drivetrain have been taken into account, thus considering the torsion dynamics of the half-shafts, the plays within the drivetrain and the relaxation length of the tyre [26]. 


\subsection{Experimental validation of the models}

An experimental activity has been carried out with a vehicle demonstrator (a front-wheel-drive sports utility vehicle) at the Lommel proving ground (Belgium).

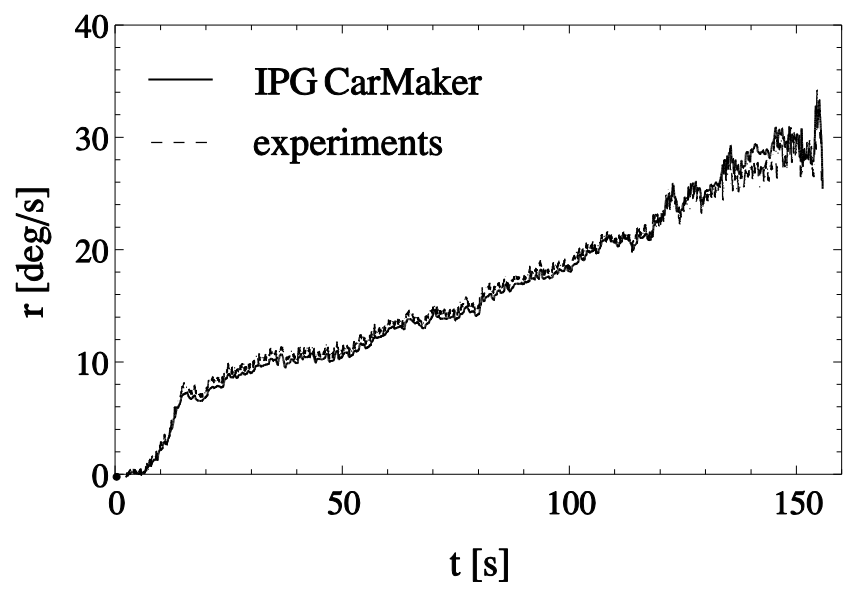

Figure 4. Yaw rate response in slowly varying conditions (skid-pad test): comparison between the results obtained from the experiments and the IPG CarMaker simulation model

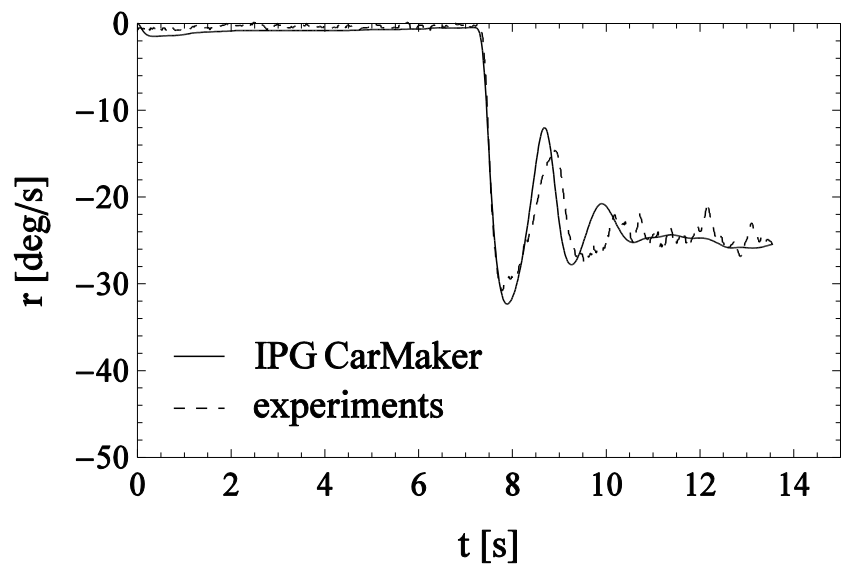

Figure 5. Yaw rate response in transient conditions (step-steer test): comparison between the results obtained from the experiments and the IPG CarMaker simulation model 
In accordance to the standards ISO4138 [27] and ISO7401 [28], skid-pad and step-steer manoeuvres were performed under a wide variety of operating conditions, i.e., selected gear, trajectory radius and vehicle velocity.

For model validation, the experimentally measured time history of the steering wheel angle and the vehicle speed were provided as inputs to the IPG CarMaker simulator. As indicated by Figures 4 and 5, during the ramp-steer and step-steer manoeuvres the yaw rate response predicted by the IPG CarMaker model matches well with the experimental measurements. The understeer and sideslip angle characteristics as functions of vehicle lateral acceleration for the test vehicle, the IPG CarMaker simulator and the quasi-static model are compared in Figures 6 and 7. The offsets related to the kinematical values of $\delta$ and $\beta$ have been subtracted from the actual values of the parameters [21]. The horizontal bars, shown for the experimental and the IPG CarMaker model results, indicate the range of variation (in terms of standard deviation with respect to the mean value) of the lateral acceleration in the time domain due to steering wheel angle oscillations that were measured during the particular manoeuvres. Owing to the good match with the experimental results, the dynamic and quasi-static models can be assumed to accurately and reliably simulate the linear and non-linear vehicle response and can be adopted as predictive tools for the evaluation of the handling response of different FEV layouts. 


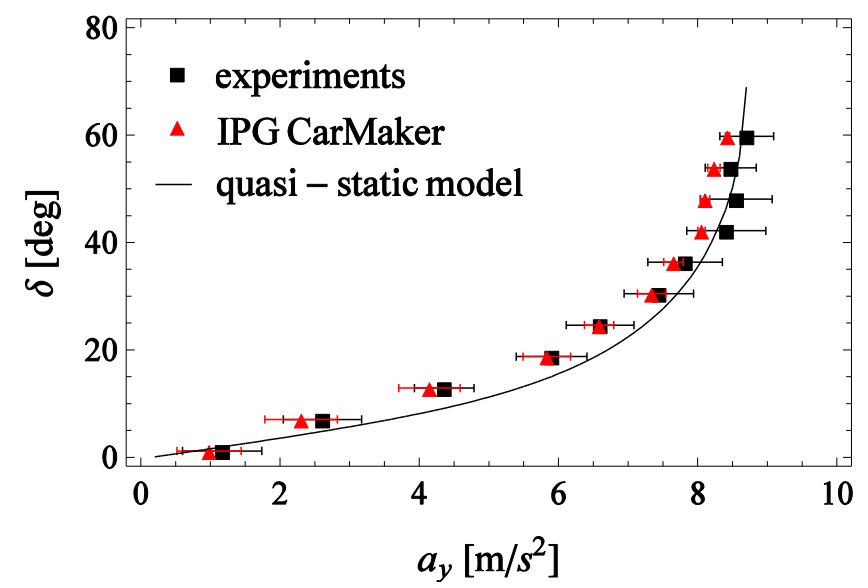

Figure 6. Understeer characteristics: steering wheel angle $\delta$ as a function of lateral acceleration $a_{y}$ for a trajectory radius of $60 \mathrm{~m}$; comparison between the experimental results, the quasi-static model and the IPG CarMaker simulation model predictions

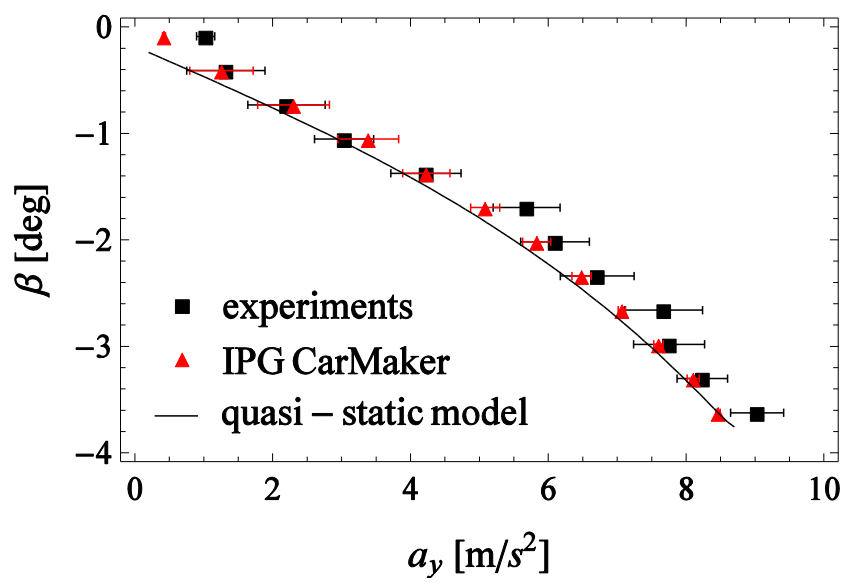

Figure 7. Sideslip angle characteristics: sideslip angle $\beta$ as a function of lateral acceleration $a_{y}$ for a trajectory radius of $60 \mathrm{~m}$; comparison between the experimental results, the quasi-static model and the IPG CarMaker simulation model predictions 


\section{The offline optimisation procedure for the design of the torque-vectoring}

controller

\subsection{Torque-vectoring and vehicle understeer}

By varying the distribution of the traction or braking torques (requested by the driver) among the driven wheels and, thus, influencing the vehicle yaw moment, $M_{z}$, the understeer behaviour of a vehicle can be significantly modified.

For instance, in trimmed conditions and disregarding tyre self-aligning moments, Equation (3) reads: $M_{z} \simeq M_{z, F_{x}}+M_{z, F_{y}}=0$, where $M_{z, F_{x}}$ is the yaw moment contribution due to longitudinal tyre forces and $M_{z, F_{y}}$ is the yaw moment contribution due to lateral tyre forces. As the intervention of the torque-vectoring controller generates a difference in longitudinal forces between the left and right wheels, $M_{z, F_{x}} \neq 0$ and, hence, $M_{z, F_{y}}=-M_{z, F_{x}}$. The condition $M_{z, F_{y}} \neq 0$ implies a variation of the lateral forces on the front and rear axles in comparison with the vehicle without torque-vectoring. In trimmed conditions, without torque-vectoring $M_{z, F_{x}} \simeq 0$ $\left(M_{z, F_{x}}\right.$ is not exactly zero due to the marginal difference between the left front and right front steering angles), from which it follows that $M_{z, F_{y}} \simeq 0$. As a consequence, if during traction tyre longitudinal forces are larger on the outer side of the corner, the lateral force on the front axle in trimmed conditions will be lower than for the vehicle without torque-vectoring at the same lateral acceleration, and the lateral force on the rear axle will be larger. As tyre lateral forces relate to tyre slip angles, the front and rear slip angles will change with respect to the vehicle without torque-vectoring. The level of vehicle understeer depends on the difference between the average front and rear slip angles of each axle, therefore the employment of torque-vectoring control allows to change the understeer characteristic of the vehicle in trimmed conditions. 
The complexity of this relationship is further increased by the interaction between longitudinal and lateral tyre forces, according to the friction ellipse. This interaction makes vehicle response sensitive to the front-to-rear torque-vectoring distribution, i.e., the front and rear axles can contribute differently to the generation of $M_{z, F_{x}}$. Therefore, the same value of $M_{z, F_{x}}$ can produce different understeer characteristics in trimmed conditions, especially when the lateral acceleration approaches its limit.

\subsection{The design specifications of the torque-vectoring controller}

As indicated by Figure 8, the understeer characteristics in traction and braking conditions can significantly vary due to the effect of the longitudinal load transfer. This variation leads to rather different vehicle turn-in behaviour which may not be predicted by the normal driver and could lead to critical driving manoeuvres. To achieve a steering behaviour that is less influenced by $a_{x}$, the feedforward part of the torquevectoring controller can be designed for specific handling targets.

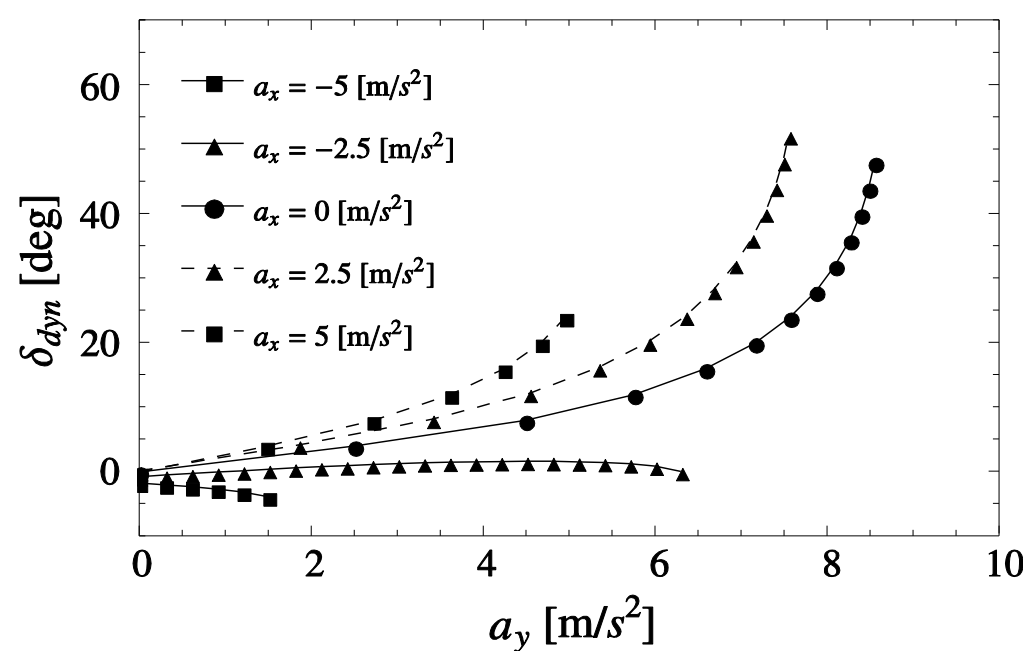

Figure 8. Understeer characteristics at a longitudinal velocity of $90 \mathrm{~km} / \mathrm{h}$ for different values of longitudinal acceleration $a_{x}$, ranging from $-5 \mathrm{~m} / \mathrm{s}^{2}$ to $5 \mathrm{~m} / \mathrm{s}^{2}$ in steps of $2.5 \mathrm{~m} / \mathrm{s}^{2}$, for a vehicle with constant wheel torque distribution in traction and braking 
(vehicle layout 2F-2R of Figure 1). Simulations results were obtained with the quasistatic model of section 2

For this study, four realistic vehicle handling targets in comparison with the same vehicle with a constant wheel torque distribution, have been set for trimmed conditions (i.e., $M_{\mathrm{z}}=0$ ). The objectives were chosen to achieve a vehicle that is predictable and easy to control to enhance vehicle safety, yet can be set up to improve agility to make the car feel sporty and direct. The handling targets are:

i) the reduction of the understeer gradient $K_{U}=\partial \delta_{d y n} / \partial a_{y}$ (where $\delta_{d y n}$ is the dynamic steering wheel angle and $a_{y}$ is lateral acceleration) in the linear part of the understeer characteristic (i.e., the part of the understeer characteristic for which the variation of $K_{U}$ is within an assigned limited percentage threshold) for $a_{x}=0$;

ii) the extension of the area of linearity of the understeer characteristic at $a_{x}=0$

iii) the increase of the maximum value $a_{y, M A X}$ of vehicle lateral acceleration;

iv) the reduction of the variation of the understeer characteristic as a function of $a_{x}$ (induced by traction and braking).

Targets i) - iii) are realised through an increase of the torque on the wheels on the outer side of the corner and a decrease of the wheel torque on the inner side. Target iv) can be achieved, for example, with torque-vectoring strategies such as those proposed in [20] and [29], which are based on traction forces $F_{x_{i}}$ distributed proportionally to tyre vertical load $F_{z_{i}}$. However, the benefit of these strategies is 
limited as the achievable extent of the reduction of the spread of the understeer characteristics cannot be predicted a-priori. Moreover, the wheel load dependence strategy is ineffective in achieving targets i) -iii) as it is not explicitly based on a reference understeer characteristic.

Hence, in order to simultaneously achieve objectives i)-iv), the novel procedure for the offline design of the feedforward torque-vectoring control action has been developed.

\subsection{The optimisation-based design of the feedforward part of the torque-vectoring controller}

The problem of designing the feedforward part of the torque-vectoring controller is addressed as an optimisation problem where a suitable objective function has to be minimised, taking into account physical constraints. The developed algorithm consists of three steps as discussed in the following three sections.

\subsubsection{Definition of the set of reference understeer characteristics}

In order to quantify the handling targets defined in section 3.2, a formulation for the reference understeer characteristic is required. Therefore, the target value of the dynamic steering-wheel angle $\delta_{d y n}$ (i.e., the difference between the actual steeringwheel angle and its kinematic value) as a function of the lateral acceleration can be defined for the relevant range of $a_{x}$. Based on the correlation with experimental data from different vehicles, a suitable analytical formulation has been found:

$$
\begin{aligned}
& a_{y}=\frac{1}{K_{U}} \delta_{d y n} \text { if } \delta_{d y n}<a_{y}^{*} K_{U} \\
& a_{y}=a_{y, M A X}+\left(a_{y}^{*}-a_{y, M A X}\right) e^{\frac{a_{y}^{*} K_{U}-\delta_{d y n}}{\left(a_{y, M A X}-a_{y}^{*}\right) K_{U}}} \text { if } \delta_{d y n} \geq a_{y}^{*} K_{U}
\end{aligned}
$$


Equation (10) defines the linear part of the reference understeer characteristic [30] and Equation (11) describes the non-linear part of the understeer characteristic, which arises from tyre saturation. The resulting function makes use of three variables that corresponds to the previously defined handling targets for vehicle cornering behaviour: the understeer gradient $K_{U}\left(a_{x}\right)$, the linear limit acceleration threshold $a_{y}^{*}\left(a_{x}\right)$ and $a_{y, \operatorname{MAX}}\left(a_{x}\right)$, which is the maximum lateral acceleration achievable by the vehicle.

\subsubsection{Definition of the problem constraints}

The physical limits of the fully electric vehicle are taken into account by setting system constraints in terms of the maximum electric motor torque and power characteristics, and the peak power of the battery pack. The combination of the physical constraints, the equations of the quasi-static model and equations (10) and (11), represent a set of equality constraints that do not fully constrain a system with two driven axles and left-to-right torque-vectoring within at least one of them. As a consequence, the minimisation of a secondary cost function is required as discussed in section 3.3.3.

\subsubsection{Definition of the objective function}

For this study, an objective function, $J_{P}$, related to the vehicle energy efficiency is used. It is based on the overall input power of the electric powertrain, $P_{T O T}$, depending on the contributions of each motor drive, $P_{\text {input }}$ : 
$J_{P}=P_{T O T}=\sum_{i=1}^{n_{\text {mot }}} P_{\text {input }_{i}}$

Hence, $M_{z, F_{x}}$ and the optimal electric motor torque distribution will be evaluated through minimisation of $J_{P}$. The algorithm which has given robust solutions of the optimisation problem for a variety of vehicle layouts and electric motor efficiency maps (usually the most critical element in the procedure [31]) is the interior-reflective Newton method [32].

\subsection{Results obtained with the optimisation procedure}

The output of the optimisation procedure is a look-up table for the wheel torque distribution as a function of steering-wheel angle, vehicle speed and accelerator/brake pedal demand. The look-up table forms the feedforward part of the reference yaw moment, $M_{z, F_{x}}$, which is added to the feedback part (zero in quasi-static conditions) as shown in the control structure in Figure 2. Within an online implementation, $M_{z, F_{x}}$ is sent to a control allocation algorithm based on the same objective function $\left(J_{P}\right)$ adopted within the offline optimisation method. This scheme has the following benefits:

i) design of the feedforward control action based on consistent control targets for any operating condition (this is not possible with simulations or experimental tests in the time domain). The set of reference understeer characteristics is also converted into the corresponding reference yaw rate look-up table for the feedback part of the controller;

ii) very quick design and critical comparison of alternative sets of achievable feedforward control actions, as the tool is computationally efficient due to the quasi-static modelling approach; 
iii) the a-priori comparison of different wheel torque control allocation techniques (by changing the objective function of the offline optimisation), without the limitations/simplifications deriving from the actual online numerical implementation of the algorithms;

iv) the a-posteriori verification of the performance of the online control allocation algorithm through its comparison with the ideal output of the offline optimisation procedure.

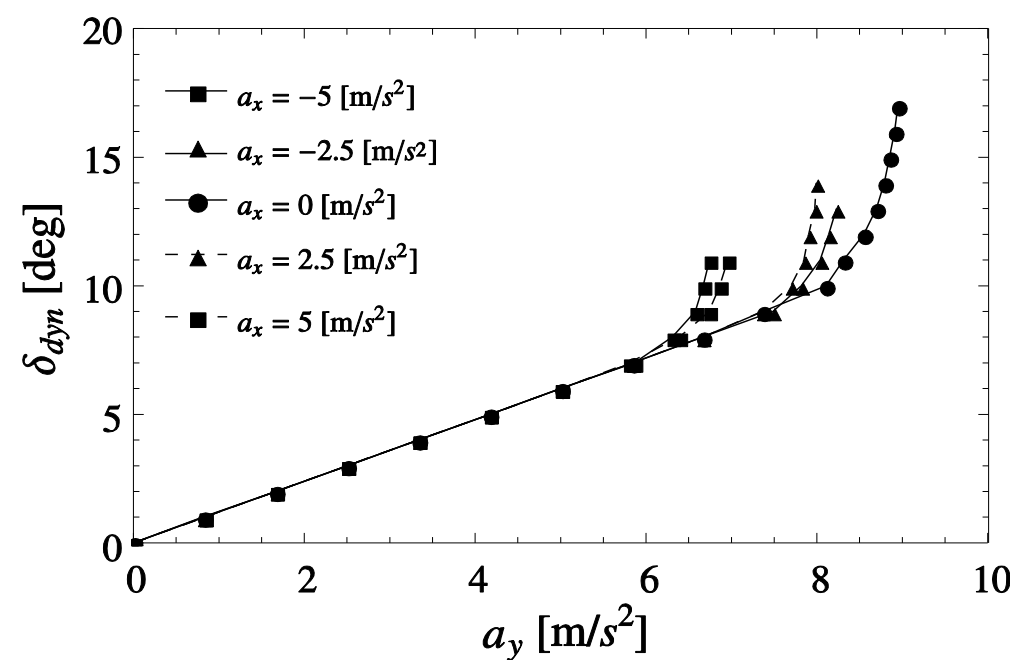

Figure 9. Understeer characteristics at a longitudinal velocity of $90 \mathrm{~km} / \mathrm{h}$ for different values of longitudinal acceleration $a_{x}$, ranging from $-5 \mathrm{~m} / \mathrm{s}^{2}$ to $5 \mathrm{~m} / \mathrm{s}^{2}$ in steps of 2.5 $\mathrm{m} / \mathrm{s}^{2}$, for a vehicle with the torque-vectoring distribution designed through the optimisation tool (vehicle layout 2F-2R of Figure 1)

As an example of the obtained results, Figure 9 plots the understeer characteristics for the same vehicle parameter set used for Figure 8, but adopting a feedforward controller designed with the optimisation procedure described above. Vehicle response is fundamentally transformed from Figure 8; it is consistent with the outlined high-level objectives of the torque-vectoring controller (see section 3.2) and 
independent from accelerator and brake pedal inputs, apart from the limited non-linear region.

The practical impact of defining a reference set of understeer characteristics is shown in Figure 10. The graph compares the simulation results obtained with the IPG CarMaker/Simulink model in terms of the response in the time domain of the vehicles of Figures 8 and 9 during a tip-in manoeuvre (i.e., fast application of a significant accelerator pedal input) carried out in cornering conditions (from the same initial $a_{y}$ ). Compared to the vehicle with a fixed wheel torque distribution, the vehicle with torquevectoring control does not show substantial variations of its yaw rate response, yielding a major benefit in terms of vehicle safety and driver's effort reduction.

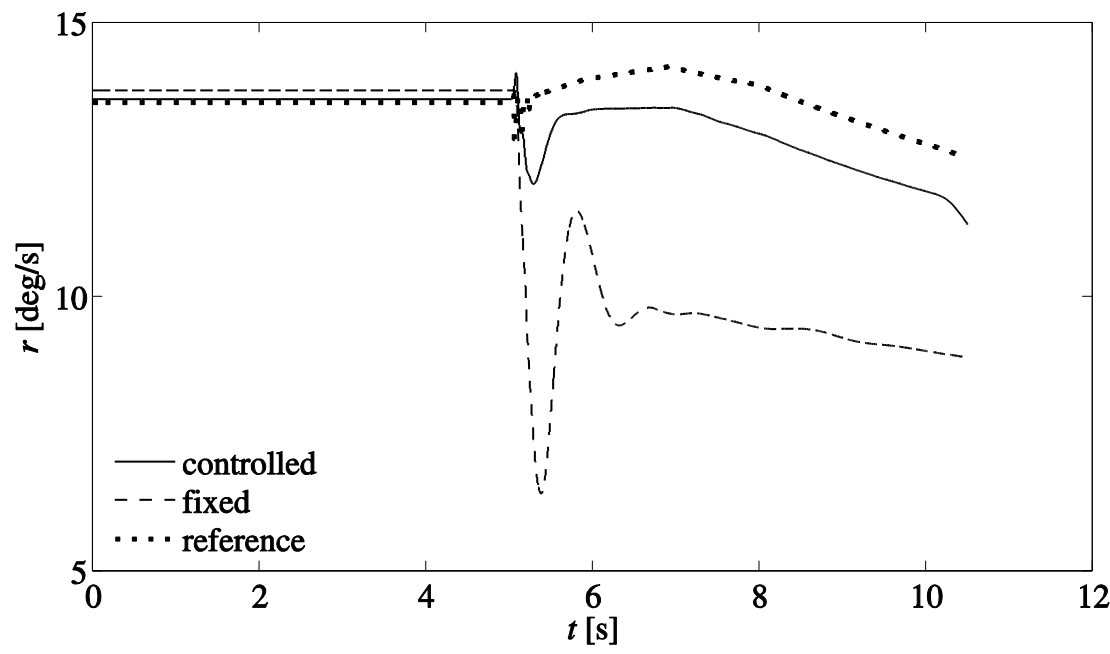

Figure 10. Tip-in manoeuvre in cornering conditions: yaw rate response of the torquevectoring controlled vehicle ('controlled'), reference yaw rate response according to the set of reference understeer characteristics of Figure 9 ('reference'), yaw rate response of the vehicle with fixed wheel torque distribution ('fixed'). Vehicle layout $2 F-2 R$ of Figure 1

\section{Evaluation metrics for different vehicle dynamic performance}

The two key challenges in the design of an electric vehicle layout with torque- 
vectoring capabilities are to understand, firstly, whether to adopt a two-wheel-drive (2WD) layout or a four-wheel-drive (4WD) layout; and, secondly, whether to adopt a torque-vectoring differential or two individually controlled motor drives for each driven axle. These choices are functions of the expected target understeer characteristics for the specific application. This section outlines how the optimisation algorithm previously described (section 3) can provide necessary information to address these two challenges. In particular, to facilitate an objective evaluation and comparison of the simulation results, three handling performance indicators $\left(I_{1}, I_{2}\right.$ and $\left.I_{3}\right)$ are introduced.

\subsection{Limit of linear vehicle behaviour (indicator $I_{1}$ )}

In order to evaluate the cornering capability of the vehicle within the linear response region, indicator $I_{1}$ is proposed. It is based on the maximum value of $a_{y}$, here called $a_{y, M A X}^{*}$, for which the vehicle can maintain a target constant $K_{U}$ at the considered value of $a_{x} \cdot a_{y, M A X}^{*}$ is derived by employing the algorithm described in section 3.3 through maximisation of the objective function (which replaces the one of Equation (12)):

$J_{A}=\sum F_{x_{i}} \sin \delta_{w_{i}}+\sum F_{y_{i}} \cos \delta_{w_{i}}$

with the main constraints being the reference linear understeer characteristic and $M_{z}=0$. Since $a_{y, M A X}^{*}$ is a function of $a_{x}$, the area of the region of the $a_{x}-a_{y}$ plan (or g-g diagram [21]) within the boundaries of the linear operating response of the controlled vehicle is proposed as performance indicator $I_{1}$ : 
$I_{1}=\int_{a_{x 1}}^{a_{x 2}} a_{y, M A X}^{*}\left(a_{x}\right) d a_{x}$

From the viewpoint of driving experience, a high $I_{1}$-value is desirable as it provides a feeling of consistency to the driver and enhances the drivers' perceptions in terms of vehicle agility and 'fun-to-drive'. In the area covered by $a_{y, M A X}^{*}\left(a_{x}\right)$ and quantified by $I_{1}$, the user will experience an 'easy-to-drive' vehicle.

\subsection{Maximum lateral vehicle acceleration (indicator $I_{2}$ )}

To examine the ultimate cornering capability of the vehicle in traction and braking conditions, performance indicator $I_{2}$ is defined based on the area covered by the graph of the achievable maximum lateral acceleration $a_{y, M A X}\left(a_{x}\right)$ [33]. In order to compute the $a_{y, M A X}\left(a_{x}\right)$ characteristics, the optimisation algorithm (section 3 ) is used to maximise the cost function $J_{A}$ (eq. 13), while considering the main constraint $M_{z}=0$ and without any condition on the steering wheel angle. Over the range of the considered longitudinal accelerations, $I_{2}$ is evaluated as:

$I_{2}=\int_{a_{x 1}}^{a_{x 2}} a_{y, M A X}\left(a_{x}\right) d a_{x}$

In terms of driving experience, $I_{2}$ will correlate with test drivers' perception of the vehicle on a test track and will be mainly useful for high performance vehicles.

\subsection{Vehicle controllability (indicator $I_{3}$ )}

The constraint to operate on an assigned set of reference understeer characteristics in quasi-static conditions is not sufficient to provide a consistent enhancement of vehicle handling performance. In fact, the reduction of vehicle understeer in traction (in order to improve fun-to-drive) usually implies a reduction of 
stability in a significant portion of the vehicle operating conditions. This interrelationship is due to the increase of lateral acceleration for the same value of steering-wheel angle, which causes larger sideslip angle and yaw rate oscillations in transient conditions. As a consequence, the controller (especially in its feedback contribution) must be capable of a significant dynamic correction of vehicle response in order to provide the expected dynamic qualities under all possible driving conditions, including transients. Vehicle controllability during steering inputs can be estimated through the evaluation of the top and bottom boundaries $\left(M_{z, M A X}\right.$ and $M_{z, M I N}$ respectively) of the achievable yaw moments as functions of vehicle sideslip angle [15]. By applying the optimisation algorithm to the quasi-static vehicle model with the cost function $J_{M}=M_{Z}$ (defined by Equation (3)) to be maximised and minimised $\forall \delta \in$ $\left[\delta_{M I N}, \delta_{M A X}\right]$, the trend of $M_{z, M A X}(\beta)$ and $M_{z, M I N}(\beta)$ defining the controllability area of $M_{z}$ can be derived.

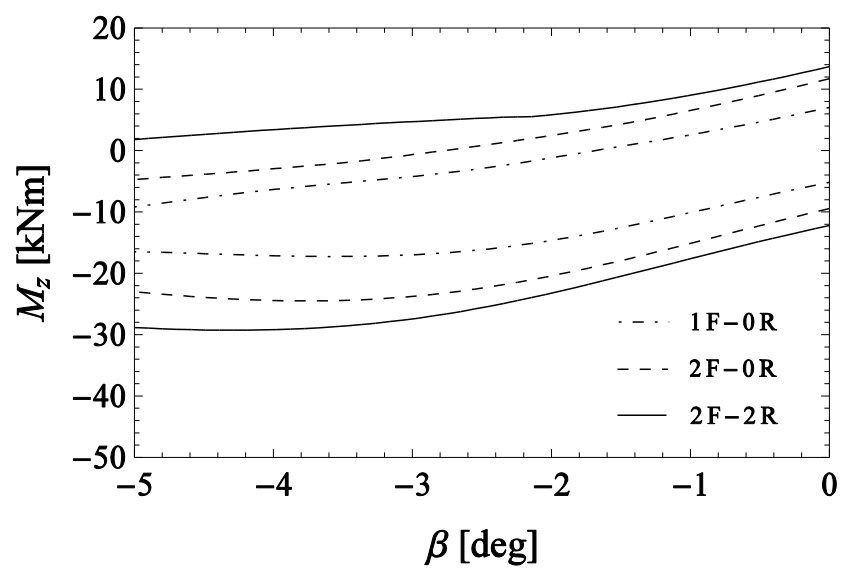

Figure 11. Comparison of the boundaries of $M_{z, M A X}$ and $M_{z, M I N}$ for vehicle layouts $1 \mathrm{~F}$ OR, 2F-0R and 2F-2R of Figure 1 (between $\beta_{M I N}=-5 \mathrm{deg}$ and $\beta_{M A X}=0 \mathrm{deg}$ ), for a range of steering wheel angles $\delta$ between -100 and $100 \mathrm{deg}$ 
Figure 11 compares the boundaries of the yaw moment plots for the vehicle layouts $1 \mathrm{~F}-0 \mathrm{R}, 2 \mathrm{~F}-0 \mathrm{R}$ and $2 \mathrm{~F}-2 \mathrm{R}$ in conditions of constant velocity $(V=90 \mathrm{~km} / \mathrm{h}) . M_{z}$ is positive when it is a destabilising moment. For the case-study vehicles with two motor drives per axle, the difference in the yaw moment controllability range $M_{Z, M A X}-M_{Z, M I N}$ is an increasing function of $|\beta|$. For the vehicle $1 \mathrm{~F}-0 \mathrm{R}$, starting from $|\beta|>3$ deg the yaw moment controllability range decreases. Moreover, at $|\beta| \approx 2 \operatorname{deg} M_{z, \text { MAX }}$ becomes negative indicating that the vehicle cannot be corrected for understeering behaviour with increasing $|\beta|$. This limitation is overcome with the $2 \mathrm{~F}-2 \mathrm{R}$ configuration because of its positive maximum yaw moment for all tested $|\beta|$. For larger values of $|\beta|$ than those included in Figure 11, the yaw moment controllability range would decrease.

Based on the area between the top and bottom boundaries (Figure 11), performance indicator $I_{3}$ is defined. To account for the stabilising and destabilising yaw moments, $I_{3}$ is composed of two parameters $I_{3, \text { Stab }}$ and $I_{3, \text { Dest }}$ relating to positive and negative $M_{z}$, respectively, along a range of sideslip angles $\left\{\beta_{M I N}, \ldots \beta_{M A X}\right\}$ :

$$
\begin{aligned}
& I_{3, \text { Stab }}=\left.\int_{M_{z, M I N}}^{M_{z, M A X}} \int_{\beta_{M I N}}^{\beta_{M A X}} d M_{z} d \beta\right|_{M_{z}<0} \\
& I_{3, \text { Dest }}=\left.\int_{M_{z, M I N}}^{M_{z, M A X}} \int_{\beta_{M I N}}^{\beta_{M A X}} d M_{z} d \beta\right|_{M_{z}>0}
\end{aligned}
$$

In particular, $I_{3, \text { Stab }}$ is an essential indicator of vehicle safety margin in transient conditions and can be adopted for predicting the potential effectiveness of the stability control system: the larger $I_{3, \text { Stab }}$ becomes, the larger is the stabilisation moment that can be applied in an emergency manoeuvre in order to restore safer driving conditions. 


\subsection{Results}

This section studies the handling performance potential of the vehicle layouts in Figure 1 on the basis of the three performance indicators defined in the previous sections. For vehicles $1 \mathrm{~F}-2 \mathrm{R}$ and $2 \mathrm{~F}-1 \mathrm{R}$, the options of a torque-vectoring differential or an open differential are included for the axle driven by a single electric motor. The torque-vectoring differential, where applicable, is modelled to provide a maximum torque bias, $\left|T_{d_{\text {Left }}}-T_{d_{\text {Right }}}\right|$, of $800 \mathrm{Nm}$. The main vehicle parameters are listed in Table 1 in the Appendix.

To ensure comparable results, the overall wheel drive torque characteristics of each tested powertrain configuration is kept equal to the one of a reference vehicle, which is currently developed in reality. The reference vehicle has a $2 \mathrm{~F}-2 \mathrm{R}$ architecture with four on-board switched reluctance electric motor drives that have been experimentally tested to derive the relevant simulation parameters. The same characteristics with one, two or three electric drivetrains have been obtained by scaling the known motor drive torque characteristics while keeping the same base speed (i.e., the speed at which the transition between the constant torque region and the constant power region of the electric motor occurs). As a result, all tested vehicle layouts have approximately the same longitudinal acceleration performance. The data for the scaled units in terms of masses and moments of inertia have been estimated in collaboration with the motor manufacturer.

The results for the performance indicators $I_{1}, I_{2}$ and $I_{3}$ have been obtained under the hypothesis that for deceleration conditions, the brake pressure for the friction brake system can be freely modulated between the two axles (as, for example, with an electrohydraulic brake system), but not within each axle, i.e., between the left and right 
callipers (as, for instance, with a vehicle dynamics control system). For traction conditions, it is stipulated that the actuation of the friction brakes is not allowed.

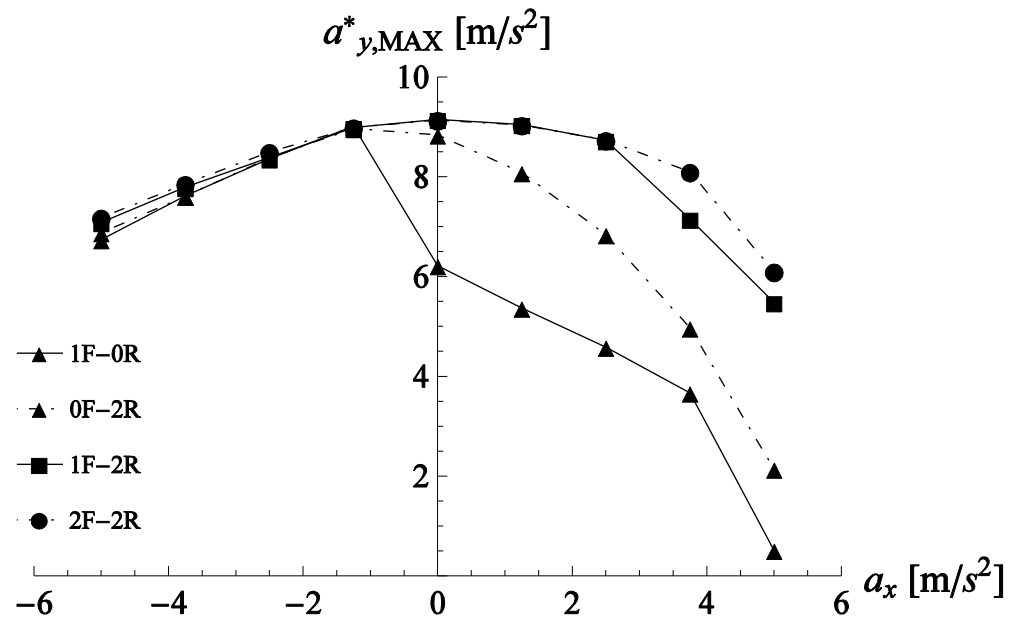

Figure 12. $a_{y, M A X}^{*}$ as a function of $a_{x}$, for the vehicle layouts $1 \mathrm{~F}-0 \mathrm{R}, 0 \mathrm{~F}-2 \mathrm{R}, 1 \mathrm{~F}-2 \mathrm{R}$ and $2 \mathrm{~F}-2 \mathrm{R}$ at $90 \mathrm{~km} / \mathrm{h}$ and with $K_{U}=10 \mathrm{deg} / \mathrm{g}$. For clarity, a reduced number of data points is shown

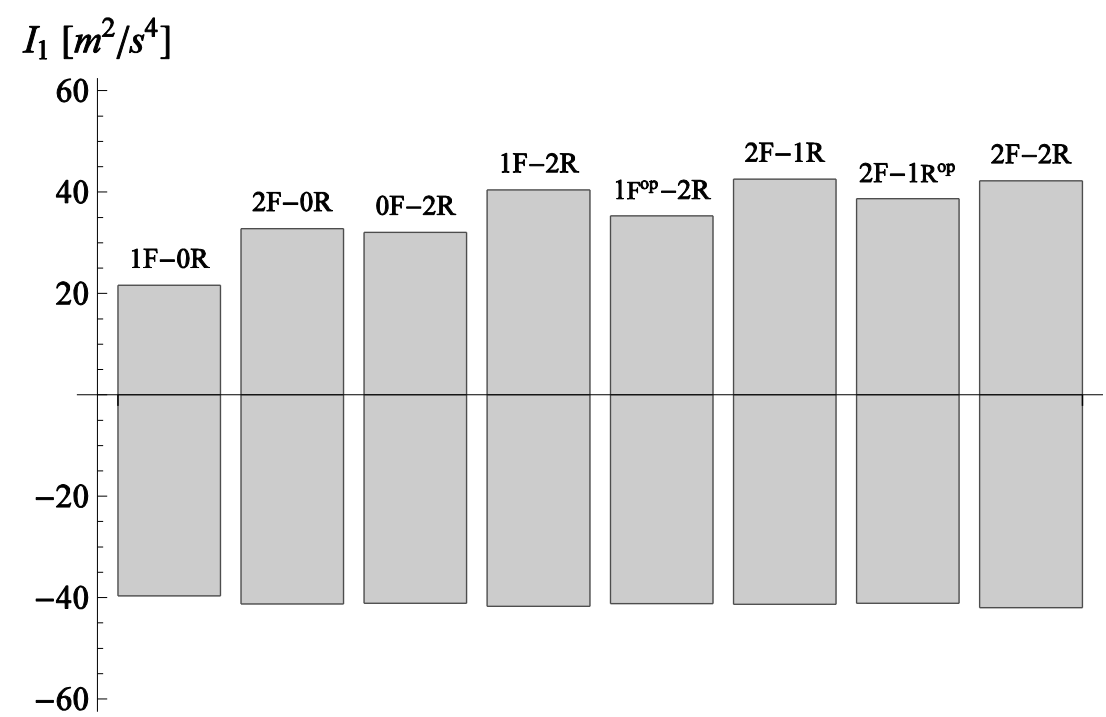

Figure $13 . I_{1}$ for the eight alternative electric vehicle layouts, at $90 \mathrm{~km} / \mathrm{h}$ and with $K_{U}=10 \mathrm{deg} / \mathrm{g}$ 
Figure 12 plots $a_{y, M A X}^{*}$ as a function of $a_{x}$ for four characteristic electric vehicle layouts. The corresponding performance indicators $I_{1}$ are shown in Figure 13. For the simulation, the reference value of $K_{U}$ for the controlled vehicles is fixed to $10 \mathrm{deg} / \mathrm{g}$, which is approximately two thirds of the $K_{U}$-value experimentally measured for the front-wheel-drive vehicle at zero longitudinal and lateral accelerations (see section 2.2). In Figure 13, the results for $I_{1}$ are shown for traction (top half of the diagram) and braking (bottom half of the diagram) conditions. As expected, the different electric vehicle layouts show almost identical results in braking due to their similar wheel brake torque capabilities achieved by the blending of friction braking and regenerative braking. In traction, the studied powertrain configurations exhibit different characteristics. Owing to the torque bias limitation, the vehicle with torque-vectoring differential (1F-0R) has a significantly reduced area of possible linear vehicle response compared to the other examined $2 \mathrm{WD}$ layouts. In traction, $I_{1}$ for vehicles $2 \mathrm{~F}-0 \mathrm{R}$ and $0 \mathrm{~F}$ $2 \mathrm{R}$ is approximately $30 \%$ higher than for vehicle $1 \mathrm{~F}-0 \mathrm{R}$. The very similar $I_{1}$ results for the rear-wheel-drive and front-wheel-drive layouts imply that the choice of drive axle does not yield advantages in terms of extension of the area of linear response for the specific case-study vehicle.

The largest $I_{1}$ values and, thus, the widest range of linear handling region can be achieved with the 4WD layouts. For example, compared to the rear-wheel-drive vehicle, $0 \mathrm{~F}-2 \mathrm{R}$, the performance indicator increases by $22 \%$ in traction conditions when the $1 \mathrm{~F}-$ $2 \mathrm{R}$ configuration is selected. This behaviour can be expected considering the wellknown safety benefits that are already achieved with modern four-wheel-drive systems of conventional cars. Yet, the proposed indicator $I_{1}$ allows to quantify the relative potential performance gain between the different layouts. 


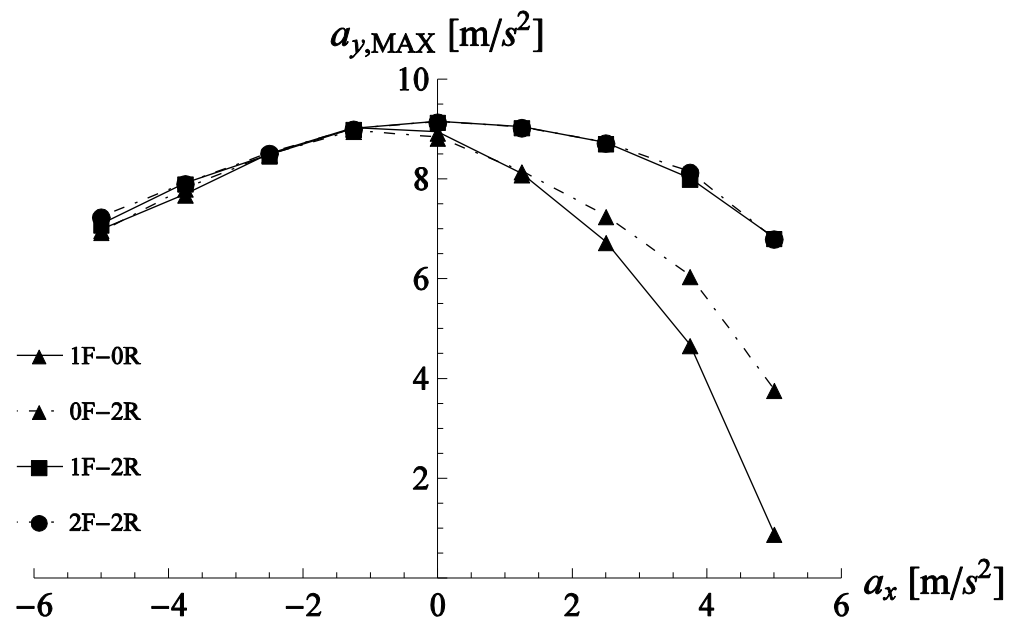

Figure 14. $a_{y, M A X}$ as a function of $a_{x}$ at $90 \mathrm{~km} / \mathrm{h}$ for the vehicle layouts $1 \mathrm{~F}-0 \mathrm{R}, 0 \mathrm{~F}-2 \mathrm{R}$, $1 \mathrm{~F}-2 \mathrm{R}$ and $2 \mathrm{~F}-2 \mathrm{R}$ with torque-vectoring control

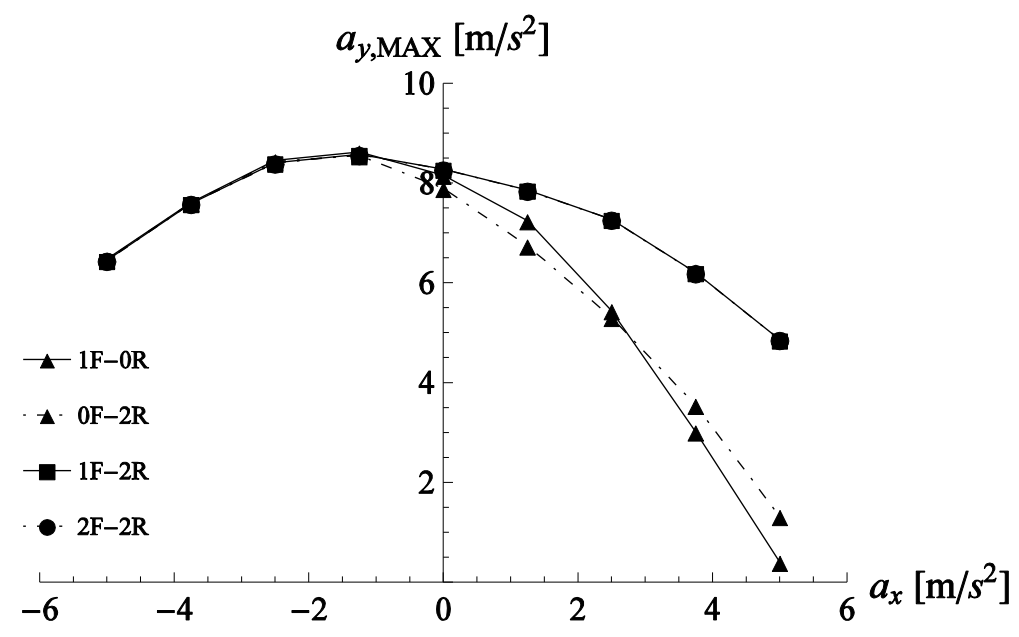

Figure 15. $a_{y, M A X}$ as a function of $a_{x}$, at $90 \mathrm{~km} / \mathrm{h}$ for the vehicle layouts $1 \mathrm{~F}-0 \mathrm{R}, 0 \mathrm{~F}-2 \mathrm{R}$, $1 \mathrm{~F}-2 \mathrm{R}$ and $2 \mathrm{~F}-2 \mathrm{R}$ without torque-vectoring control (even left-to-right torque distribution) 


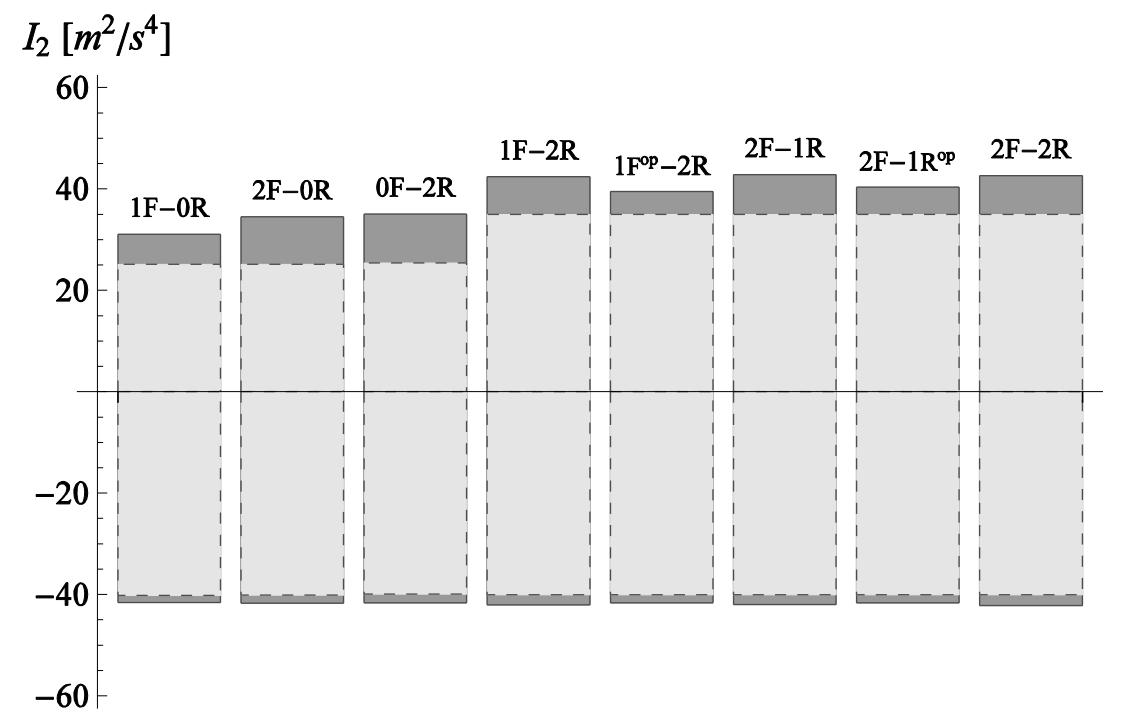

Figure 16. $I_{2}$ for the eight alternative electric vehicle layouts at $90 \mathrm{~km} / \mathrm{h}$ without (light grey) and with (dark grey) torque-vectoring control

Figures 14 and 15 plot $a_{y, M A X}$ against $a_{x}$ for the same four characteristic electric vehicle layouts, with and without torque-vectoring control. For the case of 4WD vehicles without torque-vectoring control, a fixed front-to-rear distribution (50:50 in traction and 75:25 in braking) has been used. Figure 16 shows the corresponding values of $I_{2}$, indicating the potential performance in terms of limit cornering behaviour. The uncontrolled 4WD layouts present a significant advantage over the uncontrolled 2WD layouts, i.e. approximately $27 \%$ in traction. By introducing torque-vectoring, the $2 \mathrm{WD}$ layouts can reach the lateral acceleration limits of the uncontrolled 4WD layouts, as shown by the same $I_{2}$ values. With the highest $I_{2}$ values, the $4 \mathrm{WD}$ layouts with torquevectoring control on both axles have the greatest capabilities in terms of limit cornering behaviour. Compared to the 4WD layouts with an open differential on the axle with a single motor drive, torque vectoring on two axles allows an increase in $I_{2}$ of $6 \%$ in traction. With respect to the $2 \mathrm{WD}$ layouts, the possible operating area during traction increases by $20 \%$ to $27 \%$, implying a significant extension of limit handling region. For 
vehicle $1 \mathrm{~F}-0 \mathrm{R}$, the improvement of the cornering performance achievable with torquevectoring control is marginal due to the limitation of the differential torque bias.

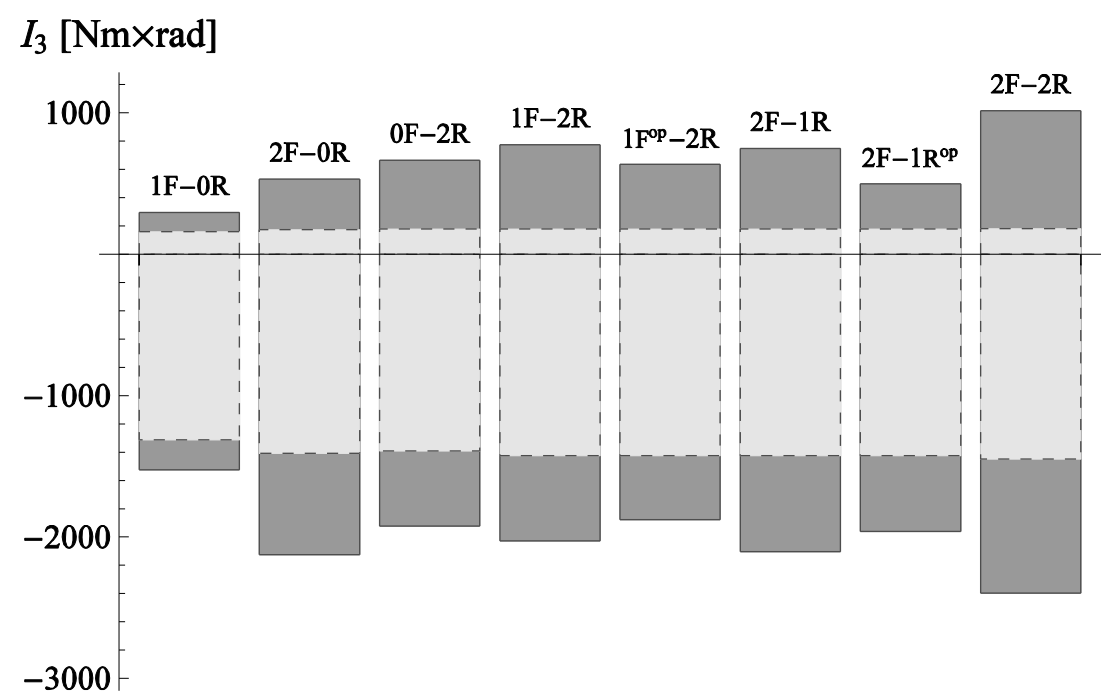

Figure 17. $I_{3, \text { Stab }}$ (negative values) and $I_{3, \text { Dest }}$ (positive values) for the eight alternative electric vehicle layouts, in conditions of constant velocity $(90 \mathrm{~km} / \mathrm{h})$

Figures 17 and 18 plot $I_{3, \text { Stab }}$ and $I_{3, \text { Dest }}$ for a vehicle at constant velocity and at $a_{x}=3 \mathrm{~m} / \mathrm{s}^{2}$ respectively. The layouts consisting of three motors and an open differential on one axle $\left(1 \mathrm{~F}^{\mathrm{op}}-2 \mathrm{R}\right.$ and $\left.2 \mathrm{~F}-1 \mathrm{R}^{\mathrm{op}}\right)$ show a benefit in terms controllability over the $2 \mathrm{WD}$ configurations only for vehicles without torque-vectoring control. When torquevectoring control is considered, the rear-wheel-drive vehicle is more effective than the front-wheel-drive vehicles (vehicles $1 \mathrm{~F}-0 \mathrm{R}$ and $2 \mathrm{~F}-0 \mathrm{R}$ ) and the layouts with three electric motors and an open differential on one axle. In contrast, the 4WD vehicle layouts with torque-vectoring control on both axles consistently provide an enhanced stabilisation capability. The vehicles with torque-vectoring differentials shows less stabilisation capabilities for dynamic steering inputs with respect to the other vehicle 
architectures as a consequence of the limitation imposed by the differential clutches on the torque bias allowable for torque-vectoring control.

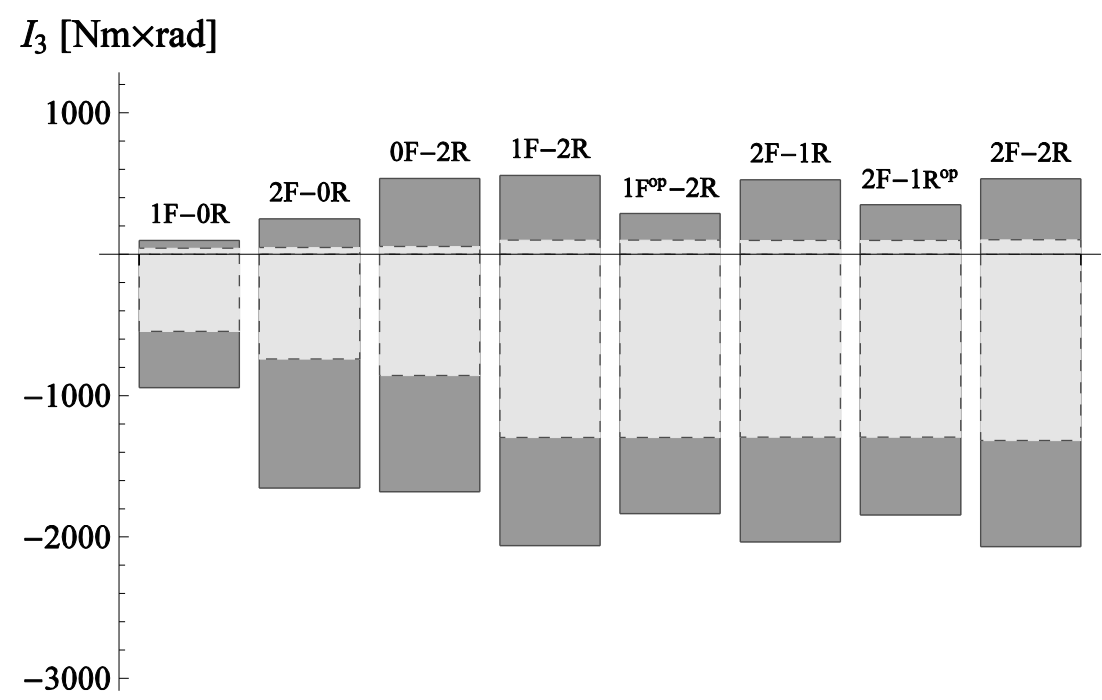

Figure 18. $I_{3, \text { Stab }}$ (negative values) and $I_{3, \text { Dest }}$ (positive values) for the eight alternative electric vehicle layouts, for constant longitudinal acceleration $\left(a_{x}=3 \mathrm{~m} / \mathrm{s}^{2}\right)$, at $90 \mathrm{~km} / \mathrm{h}$

In summary, as expected the $2 \mathrm{~F}-2 \mathrm{R}$ configuration yields the highest performing vehicle in terms of cornering behaviour. However, based on the desired specifications, the designer can easily identify other suitable configurations as shown above. Once the vehicle architecture has been selected, the engineer can re-apply the procedure with the constraints and the objective functions defined in section 3. The outcome of this step is the feedforward contribution of the controller that can be implemented, e.g., for further simulation studies in the time domain or potentially already on a prototype.

\section{Conclusions}

A novel methodology for the design of the feedforward part of the torquevectoring controller of a fully electric vehicle based on an experimentally validated quasi-static model was reported. Also, new vehicle handling performance indicators 
were proposed for the evaluation and comparison of the torque-vectoring potential of different electric drivetrain layouts. In general, the simulation results obtained reveal that:

- the quasi-static model formulation is well-suited for the quick assessment of the full range of vehicle handling capabilities;

- the offline optimisation procedure allows quick and precise development of the feedforward part of the torque-vectoring controller for a desired set of reference understeer characteristics;

- the proposed indicators facilitate an objective comparison of vehicle cornering behaviour in the linear and non-linear region, including yaw moment controllability.

With respect to the particular case study vehicle data set, the results obtained show that:

- the number of driven axles, and not their location, is the main relevant factor determining the extension of the area of linear vehicle response, which is the main perceivable parameter of vehicle handling performance for normal drivers;

- the 4WD layouts permit a tangible benefit over 2WD layouts in terms of the three handling performance indicators only when they allow torquevectoring on both axles.

\section{Acknowledgements}

The research leading to these results has received funding from the European Union Seventh Framework Programme FP7/2007-2013 under grant agreement $\mathrm{n}^{\circ} 284708$ (E-VECTOORC project). 


\section{References}

[1] A. Khaligh, Z. Li, Battery, Ultracapacitor, Fuel Cell, and Hybrid Energy Storage Systems for Electric, Hybrid Electric, Fuel Cell, and Plug-In Hybrid Electric Vehicles: State of the Art, IEEE Transactions on Vehicular Technology, Vol. 59, No. 6 (2010).

[2] M. Zeraoulia, M. El Hachemi Benbouzid, D. Diallo, Electric Motor Drive Selection Issues for HEV Propulsion Systems: A Comparative Study, IEEE Transactions on Vehicular Technology, Vol. 55, No. 6 (2006).

[3] S. Rinderknecht, T. Meier, Electric Power Train Configurations and Their Transmission Systems, International Symposium on Power Electronics, Electrical Drives, Automation and Motion, 14-16 June 2010, Pisa (Italy).

[4] B.R. Hoehn, K. Stahl, P. Gwinner, F. Wiesbeck, Torque-Vectoring Driveline for Electric Vehicles, FISITA World Automotive Congress 2012, 27-30 November 2012, Bejing (China).

[5] L. De Novellis, A. Sorniotti, P. Gruber, L. Shead, V. Ivanov, K. Hoepping, Torque-vectoring for Electric Vehicles with Individually Controlled Motors: State-of-the-Art and Future Developments, Electric Vehicle Symposium EVS26, 6-9 May 2012, Los Angeles (USA).

[6] L. De Novellis, A. Sorniotti, P. Gruber, Optimal Wheel Torque Distribution for a Four-Wheel-Drive Fully Electric Vehicle, SAE Int. J. Passeng. Cars - Mech. Syst. 6(1): 2013.

[7] D.A. Crolla, D. Cao, The impact of hybrid and electric powertrains on vehicle dynamics, control systems and energy recuperation, Vehicle System Dynamics: Vol. 50: sup1 (2012). 
[8] A. van Zanten, R. Erhardt, G. Pfaff, VDC, The Vehicle Dynamics Control System of Bosch, SAE 950749.

[9] A. Goodarzi, M. Alirezaie, A new fuzzy-optimal integrated AFS/DYC control strategy, Proceedings, 8th International Symposium on Advanced Vehicle Control (AVEC), 20-24 August 2006, Taipei (Taiwan).

[10] M. Nagai, M. Shino, F. Gao, Study on integrated control of active front steer angle and direct yaw moment, JSAE Review, Vol. 23 (2002).

[11] M. Abe, A Theoretical Analysis on Vehicle Cornering Behaviors in Acceleration and in Braking, Vehicle System Dynamics, Vol. 15 (1986).

[12] M. Nagai, Y. Hirano, S. Yamanaka, Integrated Control of Active Rear Wheel Steering and Direct Yaw Moment Control, Vehicle System Dynamics, Vol. 27 (1997).

[13] K. Shimada, Y. Shibahata, Comparison of Three Active Chassis Control Methods for Stabilizing Yaw Moments, SAE 940870.

[14] M. Graf, M. Lienkamp, Torque Vectoring Control Design Based on Objective Driving Dynamic Parameters, FISITA World Automotive Congress 2012, 27-30 November 2012, Bejing (China).

[15] A. van Zanten, Bosch ESP Systems: 5 Years of Experience, SAE 2000-01-1633.

[16] M. Canale, Vehicle Yaw Control via Second-Order Sliding-Mode Technique, IEEE Transactions on Industrial Electronics, Vol. 55, No. 11 (2008).

[17] S. Chang, T.J. Gordon, Model-based predictive control of vehicle dynamics, International Journal of Vehicle Autonomous Systems, Vol. 5, Nos. 1-2 (2007).

[18] K. Koibuchi, M. Yamamoto, Y. Fukada, S. Inagaki, Vehicle Stability Control in Limit Cornering by Active Brake, SAE 960487. 
[19] Y. Hattori, Optimum vehicle dynamics control based on tyre driving and braking forces, R\&D of Toyota CRDL, Vol. 38, No. 4 (2003).

[20] Y. Shibahata, K. Shimada, T. Tomari, Improvement of Vehicle Maneuverability by Direct Yaw Moment Control, Vehicle System Dynamics, Vol. 22 (1993).

[21] W.F. Milliken, D.L. Milliken, Race Car Vehicle Dynamics, SAE International, 1995.

[22] International Organization for Standardization (ISO), ISO 8855:2011 Road vehicles - Vehicle dynamics and road-holding ability - Vocabulary, ISO, Geneva, 2011.

[23] K. Sawase, Y. Ushiroda, T. Miura, Left-Right Torque-vectoring Technology as the Core of Super All Wheel Control (S-AWC), Mitsubishi Motors Technical Review, No. 18 (2006).

[24] L. Gao, Dynamic lithium-ion battery model for system simulation, IEEE Transactions on Components and Packaging Technologies, Issue 3, Vol. 25 (2002).

[25] http://www.ipg.de/CarMaker.609.0.html, last accessed on 25 ${ }^{\text {th }}$ April 2013.

[26] N. Amann, J. Böcker, F. Prenner, Active damping of drive train oscillations for an electrically driven vehicle, IEEE/ASME Transactions on Mechatronics, Vol. 9, No. 4 (2004).

[27] International Organization for Standardization (ISO), ISO 4138:2012 Passenger cars - Steady-state circular driving behaviour - Open-loop test methods, ISO, Geneva, 2012.

[28] International Organization for Standardization (ISO), ISO 7401:2011Road vehicles - Lateral transient response test methods - Open-loop test methods, ISO, Geneva, 2011. 
[29] S. Tanaka, M. Kawamoto, H. Inagaki, Aisin AW Co. Ltd, Regenerative braking electric vehicle with four motors, US Patent 5148833, 1992.

[30] G. Genta, Motor Vehicle Dynamics: Modelling and Simulation, World Scientific Publishing, Singapore (1997).

[31] M.J. Alexander, J.T. Allison, P.Y. Papalambros, Decomposition-based design optimisation of electric vehicle powertrains using proper orthogonal decomposition, International Journal of Powertrains, Vol. 1, No. 1 (2011).

[32] T. F. Coleman, Y. Li, An Interior, Trust Region Approach for Nonlinear Minimization Subject to Bounds, SIAM Journal on Optimization, 6 (1996).

[33] K. Sawase, Y. Ushiroda, Improvement of Vehicle Dynamics by Right-and-Left Torque-vectoring System in Various Drivetrains, Mitsubishi Motors Technical Review, No. 20 (2007).

\section{Appendix - main vehicle parameters}

Table 1. List of the main vehicle parameters for the six layouts in Figure 1.

\begin{tabular}{|l|r|}
\hline Mass [kg] & 2255 (vehicle 1F-0R); \\
& 2270 (vehicles 2F-0R and 0F-2R); \\
& 2295 (vehicles 1F-2R and 2F-1R); \\
2300 (vehicle 2F-2R)
\end{tabular}




\section{Nomenclature}

$a_{x}$ : vehicle longitudinal acceleration

$a_{x_{i}}$ : component of the wheel hub acceleration in the longitudinal direction of the wheel reference system

$a_{y}:$ vehicle lateral acceleration

$a_{y}^{*}$ : lateral acceleration threshold for linear cornering response

$a_{y, M A X}:$ maximum value of the vehicle lateral acceleration in cornering

$d_{C G}, d_{F}, d_{R}$ : heights of the roll axis, measured at the vehicle centre of gravity, the front suspension and the rear suspension

$F_{\text {drag }}:$ aerodynamic drag resistance in the vehicle longitudinal direction

$F_{T_{i}}:$ tangential force in the tyre-road contact plane

$F_{x_{i}}:$ tyre-road contact force in the longitudinal direction of the tyre reference system

$F_{y_{i}}:$ tyre-road contact force in the lateral direction of the tyre reference system

$F_{z_{i}}:$ tyre vertical force

$F_{z 0_{i}}:$ tyre static vertical force

$f_{0}, f_{1}, f_{2}$ : constant, linear and quadratic terms of the wheel rolling resistance

$I_{1}$ : performance parameter indicating the extension of the linear cornering characteristics of the vehicle

$I_{2}$ : performance parameter indicating the achievable limits of the vehicle cornering characteristics

$I_{3, \text { Dest }}:$ performance parameter indicating the destabilising contribution of the yaw 
moment diagram

$I_{3, \text { Stab }}$ : performance parameter indicating the stabilising contribution of the yaw moment diagram

$J_{A}$ : objective function related to the maximum lateral acceleration

$J_{M}$ : objective function related to the maximum and minimum yaw moment

$J_{\text {mot }_{i}}$ : electric motor moment of inertia

$J_{1_{i}}, J_{2_{i}}, J_{3_{i}}$ : moments of inertia of the primary, secondary and output transmission shafts

$J_{W_{i}}:$ wheel moment of inertia

$h_{C G}:$ vehicle centre of gravity height

$J_{e q_{i}}$ : equivalent moment of inertia of the powertrain at the motor side

$J_{H S_{i}}:$ half-shaft moment of inertia

$J_{P}$ : objective function related to the energy efficiency

$K_{U}=\partial \delta / \partial a_{y}:$ understeer gradient

$L$ : vehicle wheelbase

$m$ : overall vehicle mass

$n:$ number of drive wheels

$n_{m o t}:$ number of electric motor drives

$M_{Z}:$ yaw moment

$M_{z, F_{x}}$ : yaw moment contribution due to tyre longitudinal forces

$M_{z, F_{y}}:$ yaw moment contribution due to tyre lateral forces

$M_{z_{i}}$ : self-aligning moment at the tyre-road contact 
$M_{\varphi_{F}}, M_{\varphi_{R}}$ : front and rear anti-roll moments

$p_{i}^{r e f}$ : reference friction brake pressure

$p_{i}:$ friction brake pressure

$r^{r e f}$ : reference yaw rate

$r:$ yaw rate

$R_{i}, R_{\text {roll }_{i}}$ : laden and rolling radius of the tyre

$T_{\text {case }}:$ torque-vectoring differential carrier torque

$T_{d_{\text {Left }}}, T_{d_{\text {Right }}}$ : left and right output torques of the torque-vectoring differential

$T_{C_{\text {Left }}}, T_{C_{\text {Right }}}$ : left and right clutch torques of the torque-vectoring differential

$T_{F B_{i}}:$ friction brake torque

$T_{i}:$ wheel torque

$T_{m_{i}}^{r e f}:$ reference electric motor torque

$T_{m_{i}}:$ electric motor torque

$T_{\text {tot }}^{c}$ : overall wheel reference torque

$T_{\text {tot }}:$ sum of the wheel torques

$u$ : longitudinal component of the vehicle speed in the vehicle reference system

$V:$ modulus of the vehicle velocity vector

$V_{x_{i}}$ : component of the wheel hub velocity in the longitudinal direction of the tyre reference system

$w:$ frequency distribution of the driving cycles operating points

$x_{i}$ : longitudinal coordinate in the vehicle reference system 
$y_{i}:$ lateral coordinate in the vehicle reference system

$\alpha_{i}:$ slip angle

$\beta$ : vehicle sideslip angle

$\gamma_{i}:$ camber angle

$\delta$ : steering wheel angle

$\delta_{w_{i}}:$ wheel steer angle

$\Delta \omega_{C_{\text {Left }}}, \Delta \omega_{C_{\text {Right }}}$ : left and right slip velocities of the torque-vectoring differential clutches

$\eta_{C V 1_{i}}, \eta_{C V 2_{i}}:$ constant velocity joints efficiencies

$\eta_{1_{i}}, \eta_{2_{i}}:$ first and second reduction stages efficiencies

$\varphi$ : roll angle

$\sigma_{i}:$ slip ratio coefficient in the longitudinal direction of the tyre reference system

$\tau_{\text {Left }}, \tau_{\text {Right }}:$ torque-vectoring differential gear ratios

$\tau_{1_{i}}, \tau_{2_{i}}$ : gear ratios of the first and second reduction stages

$\omega_{\text {Left }}, \omega_{\text {Right }}$ : angular velocities of the torque-vectoring differential output shafts

$\omega_{i}:$ wheel angular speed

$\omega_{m_{i}}:$ electric motor angular speed

The superscript " ${ }^{\circ}$ ' indicates the time derivative of a variable. 